\title{
A Novel Intelligent Mechanism for Monitoring in Wireless Sensor Networks
}

\author{
Fekher Khelifi a,b, Abbas Bradai ${ }^{b}$, Med Lassaad Kaddachi ${ }^{a}$, Priyanka Rawat ${ }^{c}$ \\ ${ }^{a}$ Laboratory of Electronics and Microelectronics, University of Monastir, Monastir 5019, Tunisia \\ ${ }^{\mathrm{b}}$ XLIM Institute, University of Poitiers, Poitiers 86130, France \\ c University of Avignon, LIA-CERI, Avignon, France \\ Fakher.Khelifi@,fsm.rnu.tn, abbas.bradai@univ-poitiers.fr, lassaad.Kaddachi@isigk.rnu.tn, priyanka.rawat@univ-avignon.fr
}

\begin{abstract}
One of the main fundamental problems in designing a sensor network is to detect and locate their positions. Hence, sensors can be utilized for data collection in order to detect and organize the objects of interest. In this paper, we present a novel mechanism for object localization and detection in protected areas and surveillance zones. The goal of the proposed approach is to enhance area supervision and localize objects efficiently in wireless sensor networks.
\end{abstract}

\section{INTRODUCTION}

The scientific and industrial communities are lately showing a huge interest in wireless sensor network which consists of a set of smart sensors miniaturized with limited energy capacity [1]. Rapid deployment, reduced cost and fault tolerance in sensor networks are key features that enable deployment of different sensor network applications in various fields. There is a growing requirement for each person, for example, to be able to locate or determine whereabouts of his/her family members and relatives, or locate a service (e.g., medical help, doctor, University cafeteria, etc.). Such requirements are becoming even more significant in the context of improving the quality of life in general. Firefighters working in hazardous environments require efficient tracking systems to avoid being ending up in danger in their interventions. In hospitals, wireless sensor networks can be exploited to locate the nearest doctor of a patient, in the case of an emergency [2].

The main tasks for a surveillance system include detection and aggregation of wireless sensor network data. The network must be able to identify any object in its detection area. The most current and closest detection algorithm was able to integrate the energy values of the transmitted signals by sensors in a fixed time period [3]. If those values exceed a threshold value, the sensors indicate the presence of the detected object. This simple method suffers significant drawbacks when no objects are detected mainly if the signal consists of noise, or when the setting of the threshold is very difficult.

In [4], Ko et al. propose a detection technique of an event at the sensor node that needs to be divided into clusters. Each cluster is defined by a cluster-head which supervise, contain and detect data events that happen between the sensor nodes. A cluster-head shares information collected with his clusterheads neighbors in order to define the main algorithm threshold value.

Work in [6] surveys different surveillance systems that can detect and track patients when they have problems. These systems are based on indoor location of small nodes. As a result, these sensors communicate with each other and are able to set up some environmental parameters measurements. In addition, they can estimate the patient's position as well using less energy. However, the accuracy of the position obtained by the sensor nodes is highly dependent on the technique used. The location algorithm of the above techniques is localization using an infrared-based system. This technique is not efficient because of the non-robust infrared signal which is disrupted by the presence of daylight.

Among the known data sensing and fusion methods, a cluster network structure for WSNs. This can process the original data in the sensor nodes and reduce unnecessary transmissions. Many clustering algorithms have been specifically designed for the wireless sensor network. Among them, K-means algorithms, it organizes the sensor nodes so that each group can communicate with the fusion center with relaunched manner. In addition, clustering can also reduce bandwidth communications and routing table size stored in individual nodes[5]

In this paper, we introduce a network-based monitoring system of wireless sensors depending on an algorithm which detects and localizes objects. Our objective is to improve the detection accuracy by integrating fuzzy logic in the aggregation phase of the detected data. In addition, we focus on determining the position of the detected object. Our contributions are subsequently threefold:

1- Integration of a fuzzy logic in order to optimize data processing which is detected by the multiple type of sensors.

2- A new clustering mechanism based on the k-means algorithm which takes into account the distance between nodes for the cluster-head election process.

3- A new location mechanism based on the ultrasonic technique and finally, the implementation and operation of our system through the use of low cost hardware such as FPGAs and sensor Acoustic.

\section{II.PROBLEMATIC AND OBJECTIVES}

Monitoring of daily life activities of elderly people at home is a social problem which requires, on one hand, the need for an increased security for people living alone. On the other hand, we need to control health-related costs by delaying the entry into institution or hospitalization. The resident person must have tools that provide comfort and well-being by giving the user the ability to control his environment permanently, but also to alert in case of danger and prevent the possible risks loss. For this, solutions should be proposed to be able to monitor risky situations or possible danger (e.g., discomfort, fall, impaired balance and walking, malnutrition, poor adherence, hearing loss or vision, etc.) [7]. Some of these factors can have a significant impact with potentially serious consequences on the quality of life. It is therefore necessary to 
develop open, flexible and low-cost solutions, which are easy to install for collecting relevant data representing the life activity of the resident in his living environment. The proposed solution is based on establishing a set of wireless sensors modules with low consumption of wires, configurable and easily interfaced. Our proposal is illustrated in Figure 1.

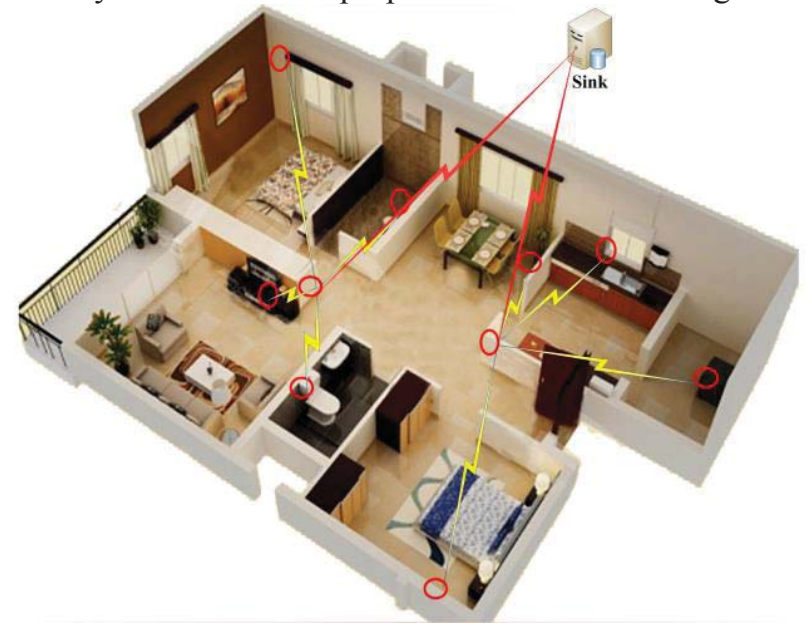

Figure 1. Scenario for Data Collection

\section{PROPOSED SYSTEM MODEL}

In this section, we consider a model of WSN based on dynamic clustering. Initially, all the nodes are deployed randomly with the same energy capacity and the same data collection process. Sensor nodes communicate with the base station via wireless links. The proposed mechanism is divided into three main phases: the detection of the object, defining its location and finally the dynamic clustering phase where multiple nodes are distributed into small groups also defined as clusters.

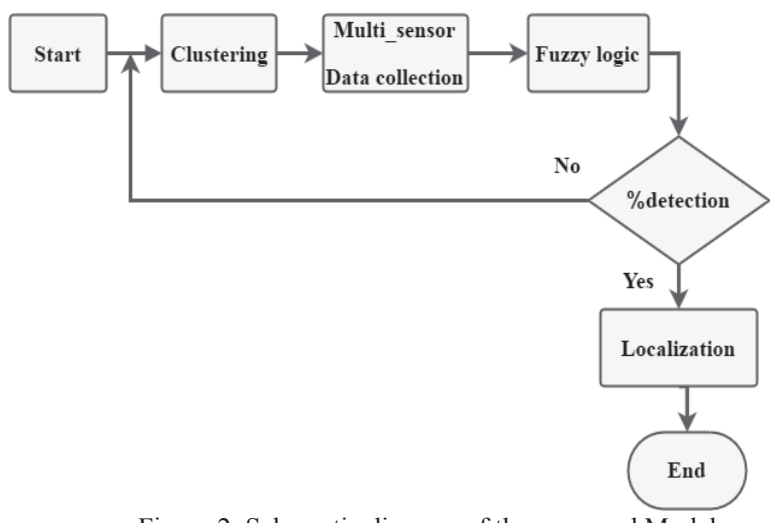

Figure 2. Schematic diagram of the proposed Model

During the detection phase, the acoustic sensors, infrared, ultrasonic are useful for detecting an object or a potential event. Each sensor detects its range during the operating period. When a sensor node detects a set of events, first, the node compares the detected value to the predefined threshold value and then triggers the transmitter to the cluster-head. Each cluster-head processes the values received by those nodes, using the fuzzy logic algorithm and sends the percentage of detection to the base station. If this percentage exceeds the threshold, the proposed system moves into the localization phase.

The localization phase of the object triggers, the moment it is detected by the sensor node. During this phase, the ultrasound sensors measure the distances using the propagation of the ultrasonic signal and time difference in radio frequency $(\mathrm{RF})$. The centroid algorithm is used to calculate the location coordinates of the detected object. The cluster-head sends these location coordinates of the detected object to the base station. When the detected value by the sensor nodes is below the threshold, the system moves into the phase of new clusters configuration.

When the network is deployed, the clusters are formed using the k-means algorithm. Each cluster has a cluster-head which processes the data collected by its members, then the detection percentage are sent to the base station. If this percentage is below the threshold, the base station selects the nodes that have non-zero values in order to form a new cluster and to verify the existence of the object. This clustering technique reduces the communication in the network and subsequently minimizes the energy consumption and as a result reduces the amount of transmitted data.

\section{CONCLUSION}

In this article, a novel monitoring mechanism is proposed for wireless sensor networks, in which we have proposed a technique for objects detection based on fuzzy logic. This technique improves the detection capability as well as increases the location accuracy using ultrasonic technology for data measurement and the centroid algorithm for data processing. To reduce the communication between nodes and the amount of data transmitted to the base station, we have proposed a novel clustering mechanism based on the k-means algorithm.

The performance analysis of our proposal has been carried out through the NS3 network simulator. Our system is realized using nexys2 FPGA which is responsible for data processing and is connected to the sensors which capture the data and send to the XBee PRO-OEM RF module for transmission

\section{V.REFERENCE}

[1] Risteska Stojkoska, B., Popovska Avramova, A., \& Chatzimisios, P.. Application of wireless sensor networks for indoor temperature regulation. International Journal of Distributed Sensor Networks, 2014

[2] Dasios, A., Gavalas, D., Pantziou, G., \& Konstantopoulos, C. (2015). Hands-On Experiences in Deploying Cost-Effective Ambient-Assisted Living Systems. Sensors, 15(6), 14487-14512

[3] M. Bahrepour, N. Meratnia, M. Poel, Z. Taghikhaki, P.J.M. Having Use of wireless sensor networks for distributed event detection in disaster management applications Int. J. Space-Based Situat. Comput., 2 (1) (2012), pp. 58-69.

[4] J.W. Ko, Y.-H. Choi A grid-based distributed event detection scheme for wireless sensor networks Sensors, 11 (11) (2011), pp. 10048-10062.

[5] Luo, Xiong, et al. "A kernel machine-based secure data sensing and fusion scheme in wireless sensor networks for the cyber-physical systems." Future Generation Computer Systems 61 (2016): 85-96..

[6] Xiao, J., Zhou, Z., Yi, Y., \& Ni, L. M. (2016). A Survey on Wireless Indoor Localization from the Device Perspective. ACM Computing Surveys (CSUR), 49(2), 25.

[7] Piau, A., Campo, E., Rumeau, P., Vellas, B., \& Nourhashemi, F. (2014). Aging society and gerontechnology: A solution for an independent living?. The journal of nutrition, health \& aging, 18(1), 97-112. 